\title{
Gonadal malignancy in patients with differences of sex development
}

\author{
Jacqueline Morin, Leslie Peard, Amanda F. Saltzman \\ Department of Urology, University of Kentucky, Lexington, KY, USA \\ Contributions: (I) Conception and design: All authors; (II) Administrative support: All authors; (III) Provision of study materials or patients: All \\ authors; (IV) Collection and assembly of data: All authors; (V) Data analysis and interpretation: All authors; (VI) Manuscript writing: All authors; (VII) \\ Final approval of manuscript: All authors. \\ Correspondence to: Amanda F. Saltzman, MD. Department of Urology, University of Kentucky, 800 Rose Street, MS 237 Lexington, KY 40536 , USA. \\ Email: afsaltzman@gmail.com.
}

\begin{abstract}
Differences of sexual development (DSD) are known to be associated with an elevated risk of malignant and pre-malignant tumors. However, given the rarity of DSD and tumors in patients with DSD, more robust, large scale, prospective literature is required to truly determine the extent of this association, long-term outcomes and the nuances associated with the wide variety of DSD diagnoses. In addition, the spectrum of diagnoses and nomenclature has been ever-changing, limiting assessment of long-term patient outcomes. This review aims to provide an overview of the pathogenesis of DSD conditions, potential malignancies associated with the diagnoses, the available screening for malignancy, and the most recent data on stratification for each DSD diagnosis and association with malignancy.
\end{abstract}

Keywords: Differences of sexual development (DSD); gonadoblastoma (Gb); germ cell tumor (GCT); seminoma; dysgerminoma

Submitted Nov 11, 2019. Accepted for publication Apr 13, 2020.

doi: $10.21037 /$ tau-19-726

View this article at: http://dx.doi.org/10.21037/tau-19-726

\section{Introduction}

The development of human gonads and genitalia is a complex process driven by multiple genes requiring expression at specific intervals during the developmental process, as well as several transcription factors and hormones allowing for gonadal differentiation (1). This process begins around 4-6 weeks gestation by the formation of urogenital ridges, derived from the mesoderm (2). Specific signaling molecules act to either activate or suppress differentiation of gonads into either testes or ovaries (3). If any factor in this process fails to act for any reason, gonadal development will be altered, leading to differences of sex development (DSD) (3). Some patients with DSD are at an increased risk of developing pre-malignant conditions such as germ cell neoplasia in situ (GCNIS) and gonadoblastoma (Gb), or malignant invasive germ cell tumors (GCT), including seminomas, non-seminomas, and dysgerminomas (4). This review aims to define the pathogenesis of malignancy in this population, review screening methods, and outline various malignancy risks based on the available literature.

\section{DSD terminology}

DSD is estimated to occur in approximately 1 in 4,5005,500 births, though the exact incidence is difficult to determine due to changes in terminology and the wide spectrum of DSD diagnoses (5). In 2005, a consensus conference was held in Chicago to discuss organization of clinical knowledge and viewpoints on patients with DSD, producing a consensus statement published in 2006 (6). One of the major accomplishments of this meeting was to define nomenclature that would more specifically categorize these patients (6). Changes included altering the terms of male or female pseudohermaphrodite and conditions of undervirilisation/undermasculinisation of XY males and 
overvirilisation/masculinization of $\mathrm{XX}$ females to $46 \mathrm{XY}$ DSD and 46XX DSD, respectively, and the term true hermaphrodite was changed to ovotesticular DSD (6). An update to the 2006 consensus statement was published in 2016 to include a more detailed guideline for workup, diagnosis, and management of these patients (5). While this update detailed the work up infants versus adolescents, addressed psychosocial/psychosexual wellbeing, and outlined an approach to gender re/assignment surgery, hormonal treatments, and fertility, the statement minimally discussed malignancy in this population (5). The statement did, however, suggest that a stratified approach could be used to identify those individuals who would require gonadectomy at time of diagnosis due to risk of malignancy development, while others may safely retain gonads for purposes of spontaneous puberty or duration of life (5).

\section{Genetic pathogenesis of DSD}

As research has expanded regarding the genes, signaling molecules, and hormones involved with sexual development, insights into how differences in this process may cause certain conditions has emerged. There are several known gene alterations that lead to one or more DSD diagnoses. While in no means an exhaustive list, the following genes have been largely implicated in the pathogenesis of DSD conditions. The Wilms tumor suppressor (WT1) gene, located on chromosome $11 \mathrm{p} 13$, plays a role in both kidney and gonadal development (3). Disorders in the function of WT1 lead to conditions such as WAGR syndrome (Wilms tumor, aniridia, genitourinary anomalies, Gb, and mental disabilities), Denys-Drash syndrome (genitourinary anomalies, Wilms tumor, and nephropathy), Frasier syndrome (gonadal dysgenesis, progressive glomerulopathy, and increased risk of Gb), and Meacham syndrome (genital anomalies, cyanotic congenital heart defects, and pulmonary hypoplasia) (3). The WNT4 gene is another gene implicated in DSD diagnoses. WNT4 acts to inhibit testes formation and support ovarian survival and development (3). This gene is associated with 46XY gonadal dysgenesis and 46XX women with atypical Mayer-Rokitansky-KüsterHauser syndrome (3). Abnormalities in the SF1/NR5A1 gene, which code for a protein involved in adrenal, gonadal, and hypothalamic development, has also been associated with a variety of DSD diagnoses (3). Mutations in the $C B X 2$ gene, which codes for a protein upstream of SRY that may act to repress ovarian development, have been found in patients with 46XY gonadal dysgenesis (3). SRY, the gene that initiates male embryonic development, is heavily implicated DSD pathogenesis, particularly in patients with 46XY gonadal dysgenesis, Turner syndrome +SRY, and patients with DSD with a variety of mosaic genotypes containing the SRY locus (3). SOX9 is a gene related to SRY that promotes Sertoli cell differentiation, and mutations of SOX9 are associated with autosomal-dominant campomelic dwarfism, gonadal dysgenesis, persistent Mullerian duct syndrome, and ovotesticular DSD (3). DAX1/NROB1 duplication is associated with gonadal dysgenesis and lossof-function mutations and is associated with $\mathrm{X}$-linked adrenal hypoplasia, which can cause cryptorchidism and hypogonadotropic hypogonadism (3). DHH plays a role in regulating morphogenesis and has been mutated in patients with gonadal dysgenesis and hypoplastic Mullerian structures (3). Three others that have been found to be mutated in patients with DSD diagnoses include GATA4, FOG2, and MAP3K1 (3). These many gene aberrancies demonstrate the complexities of the development of DSD diagnoses and provide evidence for how malignancy predisposition may be associated in this patient population.

\section{Premalignant conditions}

It is well known that some patients with a DSD are at an increased risk of malignancy (5). In general, malignancy identified at an earlier stage typically has a more favorable prognosis. There are two known premalignant conditions seen in patients with DSD-Gb and GCNIS. While Gb is a distinct type of GCNIS occurring in ovarian-type gonads, these entities can be grouped together and thought of similarly as pre-malignant conditions (Table 1).

Gb was first described by Dr. Scully in 1953 (7). This consists of a mixture of large germ cells resembling seminoma and small sex cord stromal cells, such as immature Sertoli and granulosa cells (2). Based on Dr. Scully's original 1970s data, it was estimated that $50-60 \%$ of Gb will progress to dysgerminoma or seminoma and $10 \%$ will progress to non-seminoma (8). The expression of the testis-specific protein-Y gene was the main gene implicated in development of Gb (9). It is currently hypothesized that Gb originates from germ cells initially developing along the female pathway (ovaries) but arrest in primordial follicles as they fail to complete the prophase phase of mitosis (9). There is a paucity of literature describing the natural history of $\mathrm{Gb}$ as it is generally found at the time of gonadectomy. The tumor is known to develop early in patients with certain DSD diagnoses including 46XY gonadal dysgenesis 
Table 1 Germ cell tumor histologic subtypes/analogous diagnoses

\begin{tabular}{lccc}
\hline Condition & XY without DSD & DSD & XX without DSD \\
\hline Premalignant & GCNIS & Gb/GCNIS & - \\
Malignant & Seminoma & Seminoma/dysgerminoma & Dysgerminoma \\
& Non-seminoma & Non-seminoma/non-dysgerminoma & Non-dysgerminoma \\
\hline
\end{tabular}

DSD, differences of sexual development; GCNIS, germ cell neoplasia in situ; Gb, gonadoblastoma.

and mosaic Turner syndrome with Y (10).

GCNIS is often seen in patients with DSD with testicular type tissue and it has the potential to develop into several types of testicular-type GCTs (5). This term was first introduced in 2016 in the WHO classification of urologic tumors, as a term to define precursor lesions of invasive GCTs. The use of this term helps to further categorize testicular GCTs by separating them into two groups, those derived from GCNIS, and those unrelated to GCNIS (11). GCNIS may be found in individuals with normal testes [previously intratubular germ cell neoplasia (ITGCN)]. Much like Gb, GCNIS cells are fetal gonocytes present in the seminiferous tubules that arrested during development and failed to develop to mature spermatogonia (testes) (12). The natural history of GCNIS demonstrates that these lesions often progress to an invasive GCT, but may take years to do so $(50 \%$ develop into invasive GCTs at 5 years) (13).To make matters even more confusing, both GCNIS and Gb can be present in the same gonad if there are separate testicular and ovarian elements.

\section{Learning from testicular and ovarian GCTs}

The gonads of patients with DSD are fundamentally altered and given the pathogenesis of the maldevelopment, it is intuitive that these gonads are at an elevated risk of malignancy. However, these gonadal alterations also make malignancies in patients with DSD unique and little is known about tumor behavior and prognosis in this specific patient population. The best available data right now involve loosely applying what is known about testicular and ovarian cancer in the general population, with the caveat that it is unclear if or how a DSD diagnosis may alter this information. The types of tumors seen in different gonads and their relative similarities, is summarized in Table 1.

Malignant ovarian GCTs occur at all ages of women and have an excellent prognosis as they respond well to surgical resection and cisplatin-based chemotherapy (14). Five-year cancer specific survival in women with ovarian
GCT is upwards of $94 \%$ in the general population, with a slightly lower survival rate of $92 \%$ for patients with non-dysgerminoma over pure dysgerminoma (14). This difference may be explained by the typical extensive surgical resections required in combination with chemotherapy to cure non-dysgerminoma, whereas dysgerminomas are typically very responsive to radiotherapy and chemotherapy regimens (14).

Similar to ovarian cancer, ten-year survival for testicular cancer is high, around $95 \%$ (15). This cure rate is thought to be related to the excellent responsiveness of testicular tumors to cisplatin-based chemotherapy, similar to ovarian cancer (15). This behavior of ovarian and testicular malignancies may apply to patients with DSD since the same tumors are frequently seen, particularly in patients with 46XY complete or partial gonadal dysgenesis, Turner syndrome with $\mathrm{Y}$ components, and persistent Mullerian duct syndrome (16). As such, it may be inferred that patients in the DSD population may also experience positive treatment responses and favorable prognoses despite their high risk of malignancy. However, these are merely inferences and perhaps may not be accurate, as will be detailed later.

\section{Screening}

Screening for malignancy is challenging in patients with DSD due to poor sensitivity and specificity of the currently available screening options $(17,18)$. As recommended in the general population for testicular cancer, monthly selfexamination is recommended for patients with palpable gonads (17). In patients with DSD, however, many patients do not have palpable gonads, limiting their ability to perform self-exam. In addition, the quality of evidence for sensitivity of self-exam has been noted to be low, specifically for patients with DSD (19).

Unfortunately, imaging is also poorly sensitive for identifying masses in this population $(17,18)$. In many DSD conditions, gonads are difficult to locate due to their aberrant locations, heterogenous size and appearance, and 
tendency to be mal-developed (e.g., streak gonads) (18). It is estimated that only $40-50 \%$ of gonads in DSD patients are identified with ultrasound (US) or magnetic resonance imaging (MRI), nevermind tumors in the gonads (15). US is more sensitive than MRI at identifying dysgenetic gonads, however, MRI with iv gadolinium has better sensitivity and specificity than US at localizing nonpalpable testes (15). Neither study performs well at identifying premalignant lesions, making both poor options for screening tools (15). One study reviewed imaging in patients with gonadal dysgenesis with $\mathrm{Y}$ material and found that MRI missed $100 \%$ of the malignancies in 3 patients and US identified a suspicious gonadal mass in only one of ten patients (17).

Tumor markers may also be another option for screening, although interpretation of these values must be extrapolated from the data on testicular and ovarian cancer in the general population. LDH can be associated with dysgerminoma, AFP is seen in yolk sac tumors, and $\beta$-hCG is seen in non-seminomatous GCTs, namely choriocarcinoma and some seminomas (20). However not all tumors secrete these tumor markers (i.e., teratoma, seminoma), so certainly some may be missed. McCann-Crosby et al. studied recommendations regarding screening in patients with DSD and noted although AFP, $\beta$-hCG, and LDH are associated with GCTs, there is poor evidence and unknown implications for screening in patients with DSD (19).

One of the latest advances in GCTs is the development of microRNA (miRNA) testing (21). First discovered in 2011, miRNA clusters appear to have elevated expression in the presence of GCTs and then decline to normal values after surgical removal of the tumors (12). This was the first demonstration of a screening/surveillance method more sensitive than the gold standard tumor markers and/ or imaging. GCNIS cells display specific embryonic-type miRNAs such as miR-371-3 cluster, miR-302, and miR-367 which are also expressed in invasive testicular GCTs (12). MiRNA detection studies have been focused on testicular GCTs, but recent data have demonstrated that the use of serum miRNA to accurately diagnose both seminomas and nonseminomas (but not teratomas) at the time of primary diagnosis (sensitivity $90 \%$ and specificity $86 \%$ ) (22). This recent advance provides encouragement that perhaps an easier and more accurate method of screening is on the horizon for patients with DSD.

\section{Malignancy risk categories}

Prior publications have proposed a risk stratification system based on DSD diagnoses and their risk of harboring malignancy or premalignant lesions (Table 2) (4). A recent literature review to validate this classification system demonstrated approximately $24 \%$ of patients either the high or intermediate risk category demonstrated $\mathrm{Gb}$ at gonadectomy, while $22 \%$ demonstrated invasive GCT (unpublished data). As such, it has been recommended that patients with these diagnoses undergo bilateral gonadectomy at the time of diagnosis $(19,23,24)$.

Patients of the low risk category include those with Turner Syndrome without Y, complete androgen insensitivity syndrome, 46XX ovotesticular DSD, and 46XY ovotesticular DSD (4). Though these patients did not demonstrate premalignant conditions or GCT as frequently as those in the high risk category, there was still a presence of malignancy in this group $(4,16)$.

There are DSD diagnoses that lack information regarding frequency of malignancy, placed in the unknown risk group. Recent review however shows that some diagnoses in this group have an elevated prevalence of malignancies, specifically Kleinfelter syndrome and persistent Mullerian duct syndrome (PMDS) (16). These syndromes are unique however, in that the associated malignancies are not gonadal GCTs, thus prophylactic removal is NOT advocated. Malignancy has been well described in Kleinfelter syndrome, with one study quoting a 19 times higher risk of development of mediastinal GCT as compared to the general population $(25,26)$. Despite this, the actual percentage of Kleinfelter patients developing a malignancy is roughly 1 in 4,000 (25). For patients with PMDS, the initial management usually includes leaving Mullerian remnants in place due to the low malignancy risk, however, recent case reports suggest that malignant lesions may develop in the remnants (i.e., fallopian tubes and uterus). While outside the scope of this review on GCTs, a literature review of PMDS patients over 50 years found 11 cases of Müllerian malignancy in 200 reported patients with PMDS (5\%), thus should be discussed with families when discussing removal or retention of these remnants at time of orchidopexy (27).

\section{Malignancy risk factors}

There are several well-recognized risk factors for the development of malignancy in patients with DSD. This has been advanced most recently by the explosion of genetic studies which has improved the understanding of how these conditions develop. The three major risk factors for 
Table 2 Risk stratification of DSD diagnoses (4)

\begin{tabular}{|c|c|c|c|c|c|}
\hline Risk & DSD & Malignancy risk (\%) & Recommendation & Studies (n) & Patients (n) \\
\hline \multirow{3}{*}{ High } & PAIS non-scrotal gonad & 50 & & 2 & 24 \\
\hline & Frasier syndrome & 60 & & 1 & 15 \\
\hline & Denys-Drash with $\mathrm{Y}$ & 40 & & 1 & 5 \\
\hline \multirow{3}{*}{ Intermediate } & $17 \beta-H S D$ & 28 & Monitor & 2 & 7 \\
\hline & Gonadal dysgenesis with $Y$ & Unknown & Biopsy and XRT? & 0 & 0 \\
\hline & PAIS scrotal gonad & Unknown & Biopsy and XRT? & 0 & 0 \\
\hline Low & CAIS & 2 & Biopsy and? & 2 & 55 \\
\hline \multirow[t]{2}{*}{ No (?) } & $5 A R D$ & 0 & Unresolved & 1 & 3 \\
\hline & Leydig cell hyperplasia & 0 & Unresolved & 2 & \\
\hline
\end{tabular}

?: general consensus, but further study needed. DSD, differences of sexual development.

malignancy include genomic constitution of the patient (karyotype), specifically inclusion of the $\mathrm{Y}$ chromosome, expression of embryologic germ cell markers beyond the age of $1 \mathrm{y}$, and anatomic location of gonads.

The gonadal presence and expression of the TSPY gene on the $\mathrm{Y}$ chromosome is implicated in the development of GCTs, even if the karyotype does not reveal a $\mathrm{Y}$ bearing cell line $(28,29)$. Patients with the $\mathrm{Y}$ chromosome are generally deemed high or intermediate risk (4). Importantly, a patient's peripheral blood karyotype may not correspond to his/her gonadal karyotype (30).

Prolonged expression of OCT3/4 (POU5F1) and KITL (stem cell factor) beyond 1 year of age also can play a role in malignant transformation (6). Other processes implicated in malignancy development include androgen receptor loss of function in androgen insensitivity conditions, loss-offunction mutation of MAP3K1 in 46XY gonadal dysgenesis (upregulation of the MAPK signaling pathway), loss-offunction mutations in CYP21A causing congenital adrenal hyperplasia in individuals with a 46XX karyotype, and constitutional delay of germ cell maturation in patients with trisomy 21 (24).

Abdominal gonads are at higher risk of tumor development than appropriately positioned scrotal gonads $(15,24)$. Additionally, non-palpable gonads cannot be routinely examined, either by the patient or a physician.

\section{Gonadal surgery}

The last time surgical management of patients with DSD was comprehensively updated was 2016, and as previously mentioned, malignancy risk and management were scantily discussed. In general, the consensus statement noted that a risk stratification approach could be followed to identify individuals who could either postpone gonadectomy or retain gonads for life if they are at no/low risk for malignancy development (18). Specifically, the statement recommends streak gonads in patients with XY gonadal dysgenesis, females with Y chromosome material, and patients with androgen biosynthetic defects should be removed in early childhood $(13,18)$. Despite making these recommendations, there is low quality of evidence on which these recommendations were made (19).

Gonadectomy is classically recommended to prevent invasive GCT development or allow GCT cure at an earlier stage, ideally avoiding adjuvant therapies and related risks. This strategy is very effective, but comes with a guarantee of immediate hypogonadism and infertility (31). While gonadal function is typically abnormal in patients with DSD, there may be advantages of postponed gonadectomy which are yet unrealized. For patients with complete androgen insensitivity syndrome specifically, it has been suggested that some benefits of gonadal retention may include spontaneous pubertal initiation/development, 
avoidance of issues related to poor adolescent compliance with hormonal replacement therapy, and the potential benefits of self-consent surrounding such a life-altering decision (31). As such, there must exist scientific study into the balance of the consequences and potential benefits of performing or omitting and intervention, which need to be weighed by patients and families faced with this decision. Unfortunately, widespread data addressing retention of gonads is lacking and thus the decision-making processes regarding the choice between removal or retention of gonads is unknown. The most ideal approach would be to spare and then surveil gonads in low risk patients to attempt to preserve gonadal function (both hormonal and reproductive. While certain risk factors outlined previously have been identified, proposed surveillance strategies (imaging, serum tumor markers, self-exam) have thus far been ineffective in screening) are unfortunately ineffective and thus there are no clear evidence-based guidelines to guide clinicians on how to safely and most optimal monitor retained gonads. But not only are proposed screening methods unsuccessful, the clinical impact of pre-malignant $\mathrm{Gb}$ and long-term oncologic outcomes have not been well described. This limits the ability to counsel patients and families and is an area requiring further investigation.

\section{Long-term outcomes}

The literature describes patients with DSD and malignancy, but reports are frequently single cases or small series, and long-term outcomes are scare. The largest multiinstitutional series of patients with DSD and gonadal tumors is from the Children's Oncology Group, including just 9 patients (32). This series was limited to patients with malignant ovarian non-dysgerminomas in dysgenetic gonads and compared outcomes to similar tumors in patients without DSD. Seven of the 9 identified patients had Gb associated with the malignant tumor. Three-year event free survival was $66.7 \%$ for those with DSD compared to $88.8 \%$ for those without DSD $(\mathrm{P}=0.0075)$ and 3-year overall survival was $87.5 \%$ for patients with DSD and $97.6 \%$ for those without DSD $(\mathrm{P}=0.0012)$. This suggests that patients with DSD and ovarian non-dysgerminomas have worse outcomes compared to their counterparts without DSD and perhaps these patients are a high-risk group that warrants more aggressive chemotherapy (32).

While still unpublished, a recent presentation detailing a comprehensive literature review of patients with DSD undergoing gonadal surgery described the rates of finding
Gb or GCT at the time of surgery, and also described longterm outcomes of the reported patients (16). Patients were grouped by pathologic diagnoses (GCNIS only, any GCT, no GCNIS or GCT) and compared. The Gb/GCT- and GCT-free survival by age at gonadectomy showed that in general, the rates of finding either Gb or GCT are low, but that this rate increases between 15 and 20 years of age, regardless of risk category.

With respect to oncologic outcomes, patients without $\mathrm{Gb}$ or GCT or with Gb alone have similar 5-year recurrencefree and overall survival (98-99\% and $97-99 \%$ respectively). However, those with any GCT component had significantly worse 5 -year recurrence-free and overall survival $(83.3 \%$ and $86.6 \%$ respectively). As previously described, these numbers are not quite as high as those rates reported in the literature for both testicular and ovarian GCTs found in the overall population, which is upwards of $95 \%(14,29)$.

\section{Conclusions}

Due to the inherent alteration in gonadal development associated with a diagnosis of DSD, these patients are at an elevated risk of developing gonadal malignancy. However, considering the relative paucity of the literature surrounding DSD and malignancy, the exact risk and associated risk factors remain unclear. Appropriate recommendations concerning screening have also not been well defined. At this time, we rely on extrapolating data from case reports, small series, as well as what is known in the general population with GCTs to make recommendations for screening and management in these patients.

\section{Acknowledgments}

Funding: None.

\section{Footnote}

Provenance and Peer Review: This article was commissioned by the Guest Editors (John Wiener, Jonathan Routh and Nicholas Cost) for the series "Pediatric Urologic Malignancies" published in Translational Andrology and Urology. The article was sent for external peer review organized by the Guest Editors and the editorial office.

Conflicts of Interest: All authors have completed the ICMJE uniform disclosure form (available at http://dx.doi. org/10.21037/tau-19-726). The series "Pediatric Urologic 
Malignancies" was commissioned by the editorial office without any funding or sponsorship. The authors have no other conflicts of interest to declare.

Ethical Statement: The authors are accountable for all aspects of the work in ensuring that questions related to the accuracy or integrity of any part of the work are appropriately investigated and resolved.

Open Access Statement: This is an Open Access article distributed in accordance with the Creative Commons Attribution-NonCommercial-NoDerivs 4.0 International License (CC BY-NC-ND 4.0), which permits the noncommercial replication and distribution of the article with the strict proviso that no changes or edits are made and the original work is properly cited (including links to both the formal publication through the relevant DOI and the license). See: https://creativecommons.org/licenses/by-nc-nd/4.0/.

\section{References}

1. Lucas-Herald AK, Bashamboo A. Gonadal development. Endocr Dev 2014;27:1-16.

2. Hersmus R, van Bever Y, Wolffenbuttel KP, et al. The biology of germ cell tumors in disorders of sex development. Clin Genet 2017;91:292-301.

3. Witchel S, Lee PA. Ambigous Genitalia. Pediatric Endocrinology. 4th ed. 2014.

4. Looijenga LH, Hersmus R, Oosterhuis JW, et al. Tumor risk in disorders of sex development (DSD). Best Pract Res Clin Endocrinol Metab 2007;21:480-95.

5. Lee PA, Nordenstrom A, Houk CP, et al. Global Disorders of Sex Development Update since 2006: Perceptions, Approach and Care. Horm Res Paediatr 2016;85:158-80.

6. Lee PA, Houk CP, Ahmed SF, et al, International Consensus Conference on Intersex organized by the Lawson Wilkins Pediatric Endocrine S, the European Society for Paediatric E. Consensus statement on management of intersex disorders. International Consensus Conference on Intersex. Pediatrics 2006;118:e488-500.

7. Scully RE. Gonadoblastoma; a gonadal tumor related to the dysgerminoma (seminoma) and capable of sexhormone production. Cancer 1953;6:455-63.

8. Scully RE. Gonadoblastoma. A review of 74 cases. Cancer 1970;25:1340-56.

9. Cools M, Stoop H, Kersemaekers AM, et al. Gonadoblastoma arising in undifferentiated gonadal tissue within dysgenetic gonads. J Clin Endocrinol Metab
2006;91:2404-13.

10. Bianco B, Lipay MV, Melaragno MI, et al. Detection of hidden Y mosaicism in Turner's syndrome: importance in the prevention of gonadoblastoma. J Pediatr Endocrinol Metab 2006;19:1113-7.

11. Moch H, Cubilla AL, Humphrey PA, et al. The 2016 WHO Classification of Tumours of the Urinary System and Male Genital Organs-Part A: Renal, Penile, and Testicular Tumours. Eur Urol 2016;70:93-105.

12. Rajpert-De Meyts E, Skakkebaek NE, Toppari J. Testicular Cancer Pathogenesis, Diagnosis and Endocrine Aspects. In: Feingold KR, Anawalt B, Boyce A, et al., eds. Endotext. South Dartmouth (MA): 2000.

13. Jørgensen A, Lindhardt Johansen M, Juul A, et al. Pathogenesis of germ cell neoplasia in testicular dysgenesis and disorders of sex development. Semin Cell Dev Biol 2015;45:124-37.

14. Solheim O, Gershenson DM, Trope CG, et al. Prognostic factors in malignant ovarian germ cell tumours (The Surveillance, Epidemiology and End Results experience 1978-2010). Eur J Cancer 2014;50:1942-50.

15. Pyle LC, Nathanson KL. A practical guide for evaluating gonadal germ cell tumor predisposition in differences of sex development. Am J Med Genet C Semin Med Genet 2017;175:304-14.

16. Morin J, Peard L, Vanadurongvan T, et al. Patients with Differences of Sex Development and the Development of Gonadal Malignancy - Risk Stratification and Long-term Outcomes. Presented at the Societies for Pediatric Urology Fall Congress, Scottsdale, AZ 2019, data unpublished.

17. Ebert KM, Hewitt GD, Indyk JA, et al. Normal pelvic ultrasound or MRI does not rule out neoplasm in patients with gonadal dysgenesis and $\mathrm{Y}$ chromosome material. J Pediatr Urol 2018;14:154.e1-154.e6.

18. Alaniz VI, Kobernik EK, Dillman J, et al. Utility of Ultrasound and Magnetic Resonance Imaging in Patients with Disorders of Sex Development Who Undergo Prophylactic Gonadectomy. J Pediatr Adolesc Gynecol 2016;29:577-81.

19. McCann-Crosby B, Mansouri R, Dietrich JE, et al. State of the art review in gonadal dysgenesis: challenges in diagnosis and management. Int J Pediatr Endocrinol 2014;2014:4.

20. Pressley RH, Muntz HG, Falkenberry S, et al. Serum lactic dehydrogenase as a tumor marker in dysgerminoma. Gynecol Oncol 1992;44:281-3.

21. Dieckmann KP, Radtke A, Spiekermann M, et al. Serum Levels of MicroRNA miR-371a-3p: A Sensitive and 
Specific New Biomarker for Germ Cell Tumours. Eur Urol 2017;71:213-20.

22. van Agthoven T, Looijenga LHJ. Accurate primary germ cell cancer diagnosis using serum based microRNA detection (ampTSmiR test). Oncotarget 2016;8:58037-49.

23. Hughes IA, Houk C, Ahmed SF, et al. Lawson Wilkins Pediatric Endocrine Society/European Society for Paediatric Endocrinology Consensus G. Consensus statement on management of intersex disorders. J Pediatr Urol 2006;2:148-62.

24. van der Zwan YG, Biermann K, Wolffenbuttel KP, et al. Gonadal maldevelopment as risk factor for germ cell cancer: towards a clinical decision model. Eur Urol 2015;67:692-701.

25. Williams LA, Pankratz N, Lane J, et al. Klinefelter syndrome in males with germ cell tumors: A report from the Children's Oncology Group. Cancer 2018;124:3900-8.

26. Hasle H, Jacobsen BB, Asschenfeldt P, et al. Mediastinal germ cell tumour associated with Klinefelter syndrome. A report of case and review of the literature. Eur J Pediatr 1992;151:735-9.

27. Farikullah J, Ehtisham S, Nappo S, et al. Persistent

Cite this article as: Morin J, Peard L, Saltzman AF. Gonadal malignancy in patients with differences of sex development. Transl Androl Urol 2020;9(5):2408-2415. doi:10.21037/tau-19-726
Mullerian duct syndrome: lessons learned from managing a series of eight patients over a 10 -year period and review of literature regarding malignant risk from the Mullerian remnants. BJU Int 2012;110:E1084-9.

28. Cools M, Drop SL, Wolffenbuttel KP, et al. Germ cell tumors in the intersex gonad: old paths, new directions, moving frontiers. Endocr Rev 2006;27:468-84.

29. Fosså SD, Cvancarova M, Chen L, et al. Adverse prognostic factors for testicular cancer-specific survival: a population-based study of 27,948 patients. J Clin Oncol 2011;29:963-70.

30. Ortenberg J, Oddoux C, Craver R, et al. SRY gene expression in the ovotestes of XX true hermaphrodites. J Urol 2002;167:1828-31.

31. Allen L. Opinion one: a case for delayed gonadectomy. J Pediatr Adolesc Gynecol 2009;22:381-4.

32. Dicken BJ, Billmire DF, Krailo M, et al. Gonadal dysgenesis is associated with worse outcomes in patients with ovarian nondysgerminomatous tumors: A report of the Children's Oncology Group AGCT 0132 study. Pediatr Blood Cancer 2018;65:10.1002/pbc.26913. 